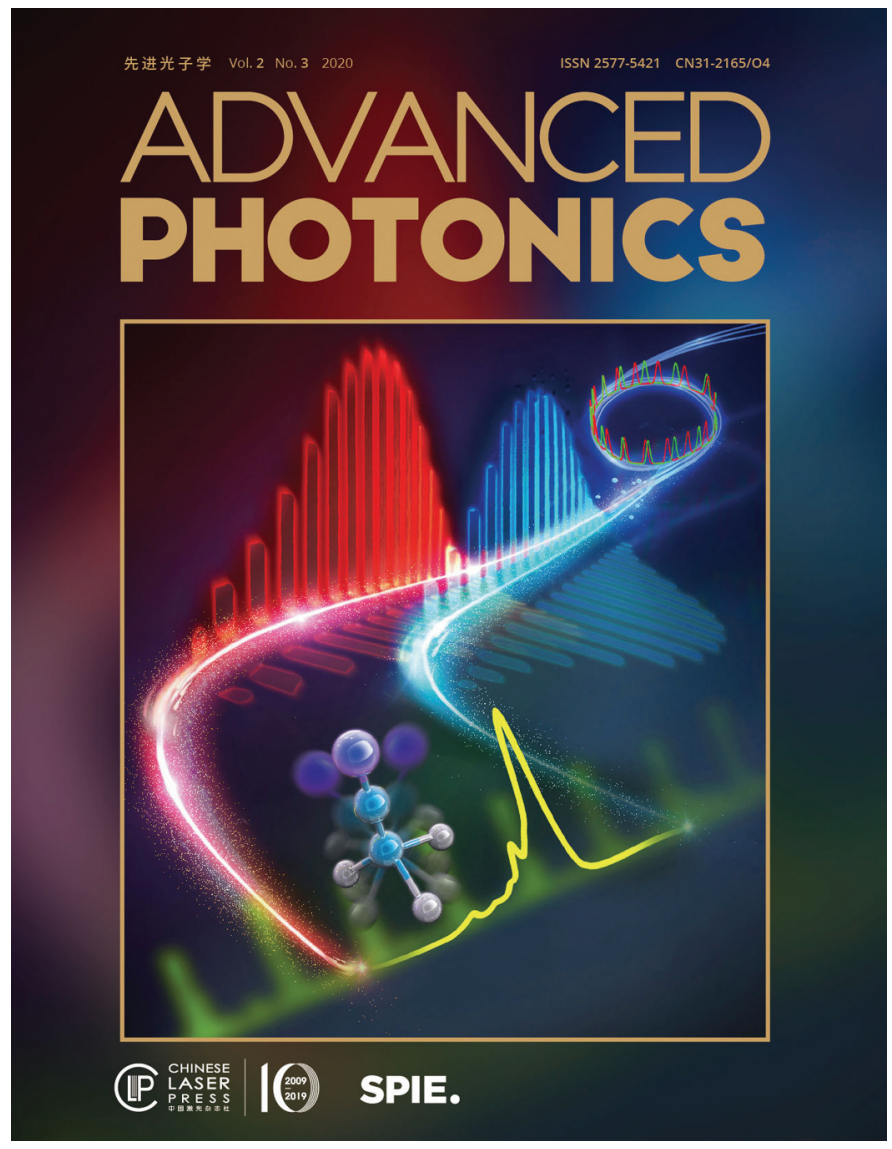

\title{
About the cover: Advanced Photonics Volume 2, Issue 3
}

The image on the cover for Advanced Photonics Volume 2, Issue 3, illustrates precise $\mathrm{THz}$ spectroscopy using a lowcomplexity dual-comb fiber laser, in which the blurry vibrational absorption spectrum of gas molecules is clearly resolved by the adaptive sampling dual-comb method. The image is based on the original research presented in the article by Jie
Chen, Kazuki Nitta, Xin Zhao, Takahiko Mizuno, Takeo Minamikawa, Francis Hindle, Zheng Zheng, and Takeshi Yasui: "Adaptive-sampling near-Doppler-limited terahertz dualcomb spectroscopy with a free-running single-cavity fiber laser," Adv. Photon. 2(3), 036004, doi: 10.1117/1.AP.2.3.036004. 